\title{
Un Néodiplostome \\ (Trematoda: Diplostomatidae) \\ chez le Tamarin \\ Leontocebus nigricollis (Spix)
}

par

\section{Georges DUBOIS}

Avec 3 figures dans le texte.

Environ 500 Leontocebus nigricollis $($ Spix $)=$ Tamarinus nigricollis (Spix) [Platyrhinia: Callitrichidae] furent piégés de 1961 à 1963 dans la jungle amazonienne supérieure, près de la frontière commune de la Colombie, du Pérou et du Brésil, puis expédiés par avion via Miami, Floride, à l'«Oak Ridge Institute of Nuclear Studies », à Oak Ridge, Tennessee, pour servir d'animaux-tests dans des études sur les radiations et l'immunologie. 11 de ces petits singes sud-américains hébergeaient dans leur intestin grêle 35 exemplaires d'une nouvelle espèce du genre Neodiplostomum Railliet. Il s'agit de la première découverte d'un Néodiplostome chez des Primates.

C'est à la demande de M. G. E. Cosgrove, de la Division Biologique de l'«Oak Ridge National Laboratory ${ }^{1}$, que nous avons entrepris l'étude d'une partie des matériaux originaux qui se répartissent comme suit:

1 Note de G. E. Cosgrove: "We are indebted to Drs. N. Gengozian and B. Nelson of the Medical Division, Oak Ridge Institute of Nuclear Studies, Oak Ridge, Tenn., for Tamarinus ». 
No d'enregistrement des hôtes

P-2554

P-2676

P-2681

P-2683

P-2753

P-2754

P-2902

P-2932

P-3057

P-3344

P-3379
Nombres de Néodiplostomes

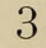

2

1

1

1

3

14

6

1

2

1

\section{Neodiplostomum (Neodiplostomum) tamarini n. sp.}

L'holotype est déposé au "Beltsville Parasitological Laboratory " MD. (U.S. Nat. Mus., Helm. Coll. no 60758). Quelques paratypes se trouvent dans la collection de l'auteur ( $\left.n^{\circ} \mathrm{R} 35-45\right)$, à l'Institut de Zoologie de l'Université de Neuchâtel; les autres sont conservés dans la collection G. E. Cosgrove.

\section{DIAGNOSE}

Segment antérieur ovale, cochléariforme, à largeur maximum au niveau de l'organe tribocytique. Segment postérieur ellipsoïde ou longuement ovoïde, un peu plus court que le précédent, à largeur maximum dans la zone testiculaire. Pore génital dorsal, subterminal. Ventouse buccale subégale à la ventouse ventrale située à milongeur du segment antérieur, parfois recouverte par le bord frontal de l'organe tribocytique circulaire, qui s'ouvre par une fente médiane. Pharynx ellipsoïdal, généralement plus petit mais plus musculeux que la ventouse buccale; prépharynx et œsophage courts; cæca atteignant presque l'extrémité du corps.

Ovaire ovoïde ou ellipsoïdal, médian ou submédian, situé transversalement ou obliquement au début du segment postérieur, dont les testicules occupent la plus grande partie. Premier testicule 


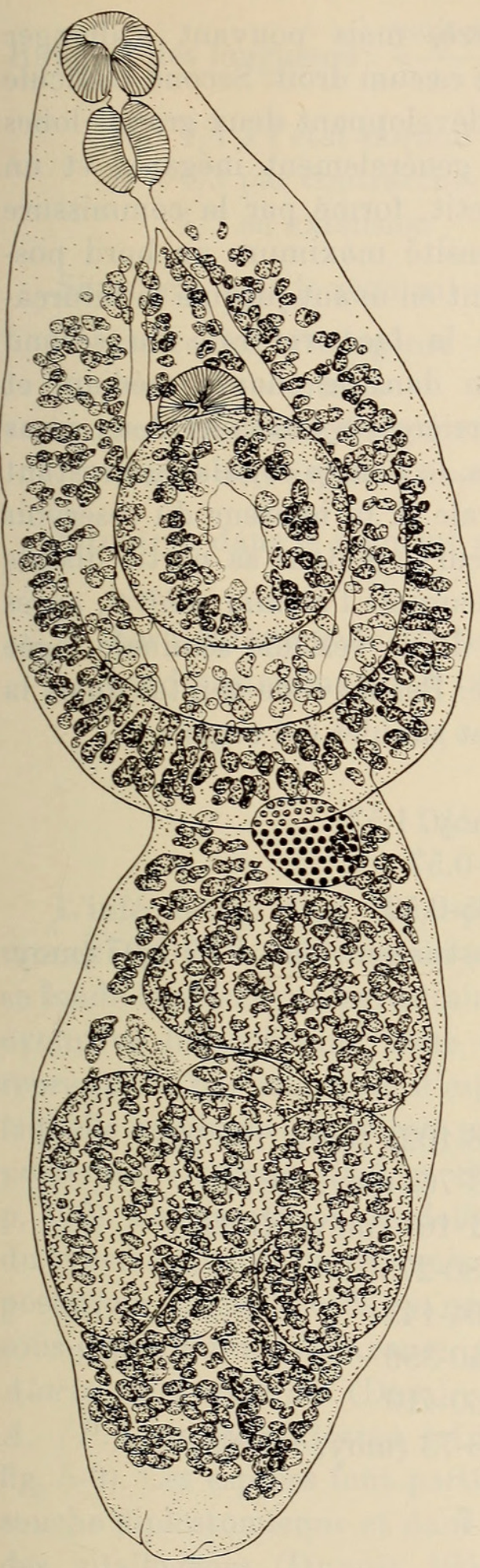

FIG. 1.

Neodiplostomum (N.) tamarini n.sp., de Leontocebus nigricollis (Spix) Holotype (P-2902)

Longueur 1,42 mm. Vue ventrale

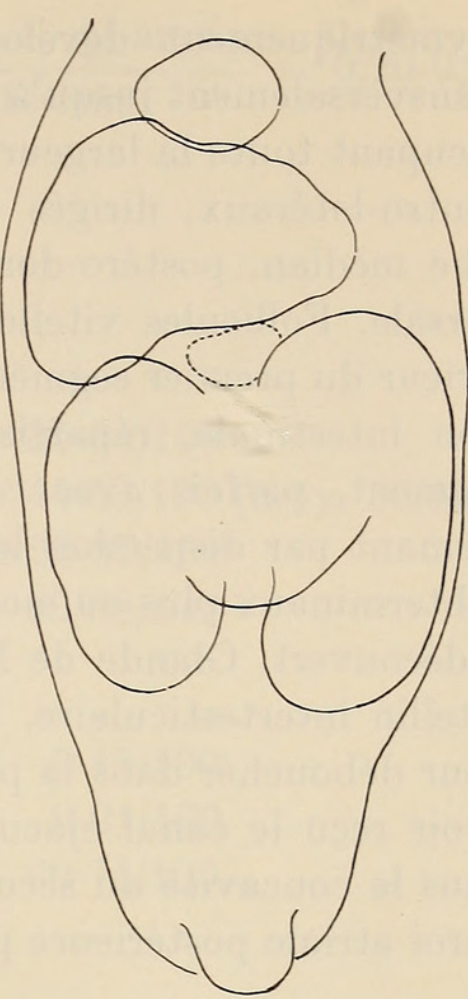

FIG. 2.

Neodiplostomum (N.) tamarini n.sp. Vue dorsale du segment postérieur Schéma topographique des glandes génitales

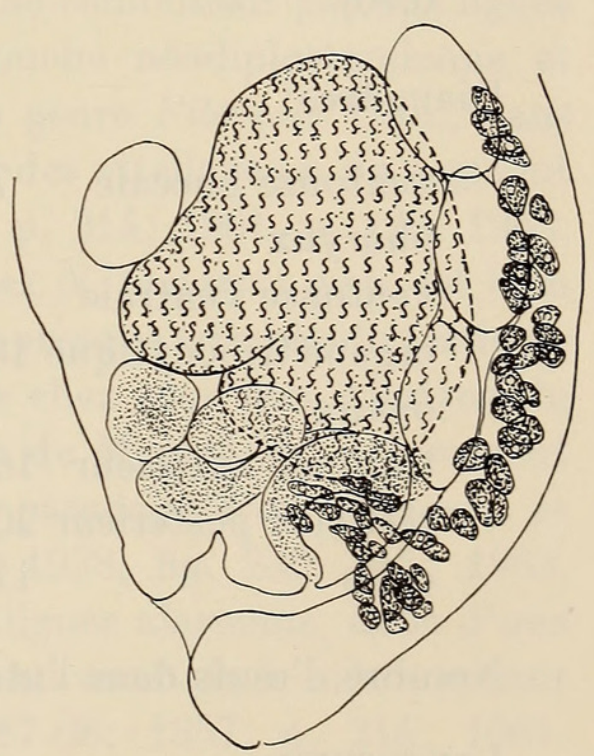

FIG. 3.

Neodiplostomum (N.) tamarini n.sp. Extrémité postérieure

(second testicule avec son lobe dorsal et un lobe ventro-latéral) 
asymétriquement développé, sénestre, mais pouvant s'allonger transversalement jusqu'à atteindre le cæcum droit. Second testicule occupant toute la largeur du corps, développant deux grands lobes ventro-latéraux, dirigés en arrière, généralement inégaux, et un lobe médian, postéro-dorsal, plus petit, formé par la commissure dorsale. Follicules vitellogènes à densité maximum au bord postérieur du premier segment, s'étendant en avant jusqu'à la bifurcation intestinale, répartis sur toute la face ventrale du second segment, parfois avec concentration dans le champ médian, et formant par dispersion latérale en arrière des testicules deux amas subterminaux plus ou moins distincts, la bourse copulatrice restant à découvert. Glande de Mehlis latérale, à la hauteur du réservoir vitellin intertesticulaire. Utérus s'incurvant dans sa partie distale pour déboucher dans la paroi postérieure de l'atrium génital, après avoir reçu le canal éjaculateur issu d'une vésicule séminale logée dans la concavité du second testicule. Pas de cône génital, mais la paroi atriale postérieure peut saillir et simuler cet organe.

Longueur totale 1,42-1,71 mm (moy. 1,53)

Segment antérieur 0,73-0,91/0,42-0,57

Segment postérieur 0,67-0,85/0,35-0,52

Rapport des longueurs: segm. post./segm. ant. 0,82-0,97 (moy. $0,90)$

Diamètres :

$\begin{array}{lc}\text { ventouse buccale } & 78-115 / 78-102 \mu(\operatorname{moy} .100 / 92) \\ \text { pharynx } & 75-84 / 63-76(\operatorname{moy} .80 / 71) \\ \text { ventouse ventrale } & 73-94 / 81-107(\operatorname{moy} .85 / 99) \\ \text { organe tribocytique } & 180-265 / 180-275 \\ \text { ovaire } & 80-99 / 104-145 \\ \text { testicule antérieur } & 160-215 / 240-350 \\ \text { testicule postérieur } & 200-325 / 260-410 \\ \text { œufs } & 112-120 / 63-73(\operatorname{moy} .115 / 68)\end{array}$

Nombre d'œufs dans l'utérus 1 à 7

Longueurs :

prépharynx 10 à $30 \mu$

œsophage 30 à 75 
Rapport des longueurs: $\frac{\text { ventouse buccale }+ \text { pharynx }}{\text { segment antérieur }}=0,20-0,31$ soit $1 / 4$ à l'état normal

$1 / 3$ par contraction

$1 / 5$ en extension

Situation dans le segment antérieur:

limite des vitellogènes

$25-34 / 100$

ventouse ventrale

44-53/100 (moy. 50/100)

bord frontal de l'organe tribocytique 50-57/100

centre de l'organe tribocytique $\quad 63-68 / 100$

Situation dans le segment postérieur:

ovaire

bord antérieur du $1^{\mathrm{e}}$ testicule

$9-21 / 100$

bord postérieur du $2^{\mathrm{e}}$ testicule

$69-74 / 100$

\section{DISCUSSION}

L'inféodation d'un Néodiplostome à un Primate constituerait une exception notable parmi les données statistiques sur lesquelles se fonde la spécificité parasitaire, si on ne connaissait pas une lignée évolutive fibricolienne, issue d'une souche néodiplostomienne et représentée par une série d'espèces du genre Fibricola Dub., dans laquelle on observe le retrait progressif des vitellogènes du segment postérieur (Duвогs 1944, p. 88; 1957, p. 214; 1961, p. 128; 1963, p. 109). La répartition des follicules chez $N$. tamarini n.sp. est bien du type néodiplostomien, mais la forme presque trilobée du testicule postérieur rappelle celle qu'on observe chez plusieurs Alariens du sous-genre Paralaria Krause, parasites de Mustélidés, notamment Alaria (P.) clathrata (Dies.), A. (P.) pseudoclathrata (Krause) et A. (P.) mustelae Bosma (cf. Du bors 1938, fig. 230, 245; 1963, fig. 5-9). Ces espèces font partie d'une lignée alarienne, issue d'une souche diplostomienne et dans laquelle on constate le même retrait des vitellogènes (Duвors 1944, pp. 87-88; 1957, p. 214; 1961, pp. $129-130 ; 1963$, p. 109). Il faut donc en conclure que le Néodiplostome du Tamarin est marqué à cet endroit par son inféodation à un Mammifère et qu'il se distingue par ce caractère de toutes les 
autres espèces du genre, chez lesquelles la gonade mâle postérieure est bilobée. De plus, les vitellogènes se dispersent latéralement en arrière des testicules, ne formant que des amas subterminaux peu denses ou même à peine distincts, ce qui n'est pas le cas des autres espèces congénériques, où des condensations de follicules apparaissent de chaque côté de la vésicule séminale.

Le rapport des longueurs : ventouse buccale + pharynx/segment antérieur semble discriminatif par sa valeur élevée (1/3 à 1/5): il caractérise bien la nouvelle espèce.

Nous remercions M. G. E. Cosgrove de nous avoir confié la détermination de ce parasite.

\section{RÉSUMÉ}

Neodiplostomum (N.) tamarini n.sp. a été trouvé chez 11 spécimens de Leontocebus nigricollis (Spix) [Platyrhinia].

\section{BIBLIOGRAPHIE}

Dubois, G. 1938. Monographie des Strigeida (Trematoda). Mém. Soc. neuchâtel. Sci. nat. 6: 1-535.

-- 1944. A propos de la spécificité parasitaire des Strigeida. Bull. Soc. neuchâtel. Sci. nat. 69: 5-103.

-_ 1957. La spécificité de fait chez les Strigeida (Trematoda). Premier Symposium sur la spécificité parasitaire des parasites de Vertébrés: 213-227, Neuchâtel.

-- 1961. A propos de Neodiplostomum Railliet 1919 et Fibricola Dubois 1932 (Trematoda: Strigeida). Bull. Soc. neuchâtel. Sci. nat. 84: 125-132.

-- 1963. Statut des Alariinae Hall et Wigdor 1918 (Trematoda: Diplostomatidae) et revision de quelques Alariens. Ibid. 86: 107-142. 


\section{$2 \mathrm{BHL}$ Biodiversity Heritage Library}

Dubois, Georges. 1966. "Un Néodiplostome (Trematoda: Diplostomatidae) chez le Tamarin Leontocebus nigricollis (Spix)." Revue suisse de zoologie 73, 37-42. https://doi.org/10.5962/bhl.part.75806.

View This Item Online: https://www.biodiversitylibrary.org/item/126811

DOI: https://doi.org/10.5962/bhl.part.75806

Permalink: https://www.biodiversitylibrary.org/partpdf/75806

\section{Holding Institution}

Smithsonian Libraries

\section{Sponsored by}

Biodiversity Heritage Library

\section{Copyright \& Reuse}

Copyright Status: In Copyright. Digitized with the permission of the rights holder.

Rights Holder: Muséum d'histoire naturelle - Ville de Genève License: http://creativecommons.org/licenses/by-nc-sa/3.0/

Rights: https://www.biodiversitylibrary.org/permissions/

This document was created from content at the Biodiversity Heritage Library, the world's largest open access digital library for biodiversity literature and archives. Visit BHL at https://www.biodiversitylibrary.org. 